\title{
Different Recurrence and Growth Pattern of Gliomatous Foci after Mesenchymal Stem Cell Harbouring the Herpes Simplex Thymidine Kinase Gene Transplantation in Multifocal Glioblastoma Multiform: A Clinical Case Report
}

Saeed Oraee Yazdani ( $\square$ Saeed_o_yazdani@yahoo.com )

Shahid Beheshti University of Medical Sciences School of Medicine https://orcid.org/0000-0003-45509698

\section{Mohammadhosein Akhlaghpasand}

Shahid Beheshti University of Medical Sciences School of Medicine

Maryam Golmohammadi

Shahid Beheshti University of Medical Sciences School of Medicine

\section{Fatemeh Rostami}

Islamic Azad University Science and Research Branch

\section{Gelareh Shokri}

Stem Cell Research Center

Maryam Hafizi

Development and Research Center of State Council Department of Development Strategy and Regional Economy

\section{Maryam Oraee-Yazdani}

Shahid Beheshti University of Medical Sciences School of Medicine

\section{Ali-Reza Zali}

Shahid Beheshti University of Medical Sciences School of Medicine

\section{Masoud Soleimani}

Tarbiat Modares University

\section{Short report}

Keywords: Glioblastoma multiform (GBM), Mesenchymal Stem Cells (MSCs), HSV-TK, magnetic resonance imaging (MRI)

Posted Date: June 1st, 2021

DOI: https://doi.org/10.21203/rs.3.rs-556539/v1 
License: (c) (i) This work is licensed under a Creative Commons Attribution 4.0 International License. Read Full License 


\section{Abstract}

Background: Glioblastoma multiform (GBM) as a malignant brain tumor has poor prognosis despite the current therapies. Suicide gene therapy is one of the most widespread cancer gene therapies which requires mRNA encoding a pro-drug activating enzyme transduced into the vector such as Mesenchymal Stem Cells (MSCs) that injects into the tumor tissue and leads to tumor suppression.

Case presentation: In this case report, the authors describe a 37-year-old man with two obvious foci of glioblastoma multiform at left frontal and left parietal lobes. After the resection of the foci, the patient received stem cell-mediated herpes simplex virus thymidine kinase (HSV-TK) gene at frontal focus of tumor besides ganciclovir as prodrug for 14 days after injection. Then he was followed up with regular magnetic resonance imaging (MRI). The growth and recurrence pattern of foci was assessed. After the injection on Feb 09, 2019, the patient's follow-up revealed recurrence of parietal focus on Dec 19, 2019; however, frontal focus had a slight and unremarkable enhancement. Until our last radiological follow-up (on Mar 18, 2020) left frontal focus had no prominent recurrence and the size of the left parietal focus increased and extended to the contralateral hemisphere through the corpus callosum. Eventually patient has passed away on July 16, 2020 (PFS=293 days, OS=657 days).

Conclusions: It seems that the gliomatous focus that undergone administration of bone marrow MSC containing the HSV-TK gene, had a different pattern of growth and recurrence compared to a non-treated one.

\section{Introduction}

Glioblastoma multiform (GBM), as the most common malignant brain tumor in adults, has an average survival time of fewer than 12 months despite current therapies (1). The incidence of multiple synchronous GBM lesions in the same patient is reported between $0.5 \%$ and $35 \%$ of diagnosed GBM cases with an average of $~ 10 \%(2)$.

According to recent clinical trials, suicide gene therapy is one of the most widespread cancer gene therapies with encouraging results (3). The most widely approach used in gene therapy studies on glioma is the herpes HSV-TK that phosphorylate nucleoside analogs such as ganciclovir (GCV) to suppress DNA replication of cells (4).

In gene therapy, the efficient delivery of the gene to the target and gene expression is one of the most major issues (5). In addition to viral vectors, applying MSCs as a vehicle has a special place in cancer cell therapy (6). It is now well accepted that the MSCs tropism to inflammatory sites and microenvironment of the tumor made these cells very attractive for targeting of genes to tumor sites (7).

In this study, we report a case of multifocal glioblastoma multiforme with gliomatous foci in the left frontal and left parietal lobes. Following the resection of the second focus, we treated the left frontal focus with MSC-HSV-tk / GCV approach. To the best of our knowledge, this is the first report, investigating 
and well presenting the different recurrence and growth patterns of treated gliomatous foci with MSCHSV-tk / GCV approach in comparison with control gliomatous foci in one patient.

\section{Case Presentation}

A 37-year-old man (Karnofsky Performance Score-KPS > 90) with multifocal glioblastoma multiform referred to the outpatient neurosurgery clinic of Shohadaye-Tajrish hospital on Oct 31, 2018. He had two gliomatous foci which were in the left frontal and left parietal lobes. Left parietal focus had been undergone resection at another centre 35 days ago. Another lesion at the frontal lobe was resected at our centre through frontal craniotomy on Nov 5, 2018. Pathology of both foci revealed that these tumors were grade IV astrocytoma (glioblastoma multiforme). According to the immunohistological and PCR tests, both foci had wild type of IDH1 and IDH2, and the promotor of MGMT was methylated under 5 percent. Standard radiation therapy was started on Nov 25, 2018 following Temozolomide ( $75 \mathrm{mg} / \mathrm{m} 2 /$ day) was taken one hour before radiation therapy for the first week and then continued separately until 54 days until Jan 20,2019. After the accomplishment of radio/chemotherapy, regarding the eligibility of the patient, he volunteered for participation in our stem cell-mediated gene therapy of glioblastoma program. This project was designed in accordance with the declaration of Helsinki and was approved by the Ethics committee in the Medical Research of the Shahid Beheshti University of Medical Sciences; license number: IR.SBMU.REC.1396.224 (Clinical trial registration code: IRCT20200502047277N2). All procedures were performed after written informed consent was obtained. The patient was fully aware of the experimental process of the treatment, unexpected outcomes, and possible adverse effects.

Collection of MSCs was performed in the operating room under sterile conditions. The patient was discharged after the procedure on the same day. Bone marrow blood $(100 \mathrm{ml})$ was aspirated from the iliac bone for the collection of bone marrow stem cells according to the protocol that we used in our previous study and we assessed the MSCs characteristics through analysing the cell surface markers such as CD90, CD105, CD45, and CD73 (8).

Human embryonic kidney (HEK) 293 cell lines were obtained from Iranian Biological Resource Centre and cultured in high-glucose Dulbecco's modified Eagle medium (DMEM, Gibco, USA) containing 10\% Fetal Bovine Serum (FBS, Gibco, USA) and 1\% nonessential amino acids (Invitrogen, USA), and L-glutamine (2 $\mathrm{mM}, \mathrm{Gibco}, \mathrm{USA})$. Cells were cultured under a standard condition in $95 \%$ humidity and $5 \% \mathrm{CO} 2$ at $37^{\circ} \mathrm{C}$.

For the gain of function study, thymidine kinase was cloned into the pCDH-CMV-MCS-EF1-copGFP plasmid that was previously digested with $\mathrm{BamHI}$ and EcoRI endonuclease enzymes. For lentivirus production, HEK-293T cells were calcium phosphate co-transfected with pCDH- Thymidine kinase, pSPAX2 plasmid (packaging plasmid Contains Gag, Pol, Rev, and Tat) and pMD2 plasmid (containing vesicular stomatitis viruses (VSVs) glycoprotein (G)). The supernatants were harvested every 12 hours for 3 days after transfection and concentrated by ultracentrifuge at $47,000 \times \mathrm{g}$ for 2 hours at $4^{\circ} \mathrm{C}$. To induce thymidine kinase expression in MSCs; MSCs were cultured in DMEM/F12 and transduced with $\mathrm{PCDH}-$ thymidine kinase lentivirus. $3 \times 10^{5}$ cells were transferred to a T25 flask and transduced the following day 
with Multiplicity of infection (MOI) 40 (TU/cell). Also, transduced cells with pCDH-TK were selected by puromycin $(2 \mu \mathrm{g} / \mathrm{ml})$. After purification of transducing cells by puromycin, they were washed three times with normal saline afterwards; the cells were ready for injection.

Human MSCs highly expressed CD90 and CD73, and were positive for the CD105 marker. Furthermore, the expression of CD45 observed in low percentage of these cells.

After virus production and concentration, the virus titration was determined by the use of flow cytometry. Following the titration of recombinant viruses, the mesenchymal stem cells were transduced with a certain amount of the virus (MOI 40) (Fig. 1).

The patient was hospitalized and underwent general anaesthesia and a direct injection of MSCs transduced with lentivirus was given through the previous frontal craniotomy under the guidance of navigation in the bed of the frontal tumor. The patient received $5 \times 10^{5} \mathrm{MSC}$ transfected by lentivirus containing HSV-TK enzyme in $1 \mathrm{cc}$ volume via the injection catheter which had been connected to the navigation system before using. The catheter was placed at the site of the injection for one minute to decrease leakage. Forty-eight hours after cell injection, the patient received intravenous ganciclovir with a dose of $5 \mathrm{mg} / \mathrm{kg}$ over 1 hour, every $12 \mathrm{~h}$. The drug administration continued for a total of 28 doses over 14 days.

The patient was followed up after injections and during the prodrug therapy and assessed for any adverse effects based on common terminology criteria for adverse events (CTCAE, version 4.3). Blood and urine samples were analysed during hospitalization to control hematologic toxicity and renal impairment due to ganciclovir medication.

Radiological recurrence was assessed by MRI, obtained before injection, and then every 3 months and checked independently by two neurosurgeons. This assessment was done by MRI according to Response Evaluation Criteria in Solid Tumors (RECIST) where progression was defined as at least a $20 \%$ increase in the sum of the longest diameters of the target lesions.(9)

After the cell injection, our patient was in a good general state and he was conscious and comfortable. The patient had prior history of seizures, but due to the lack of regular medication, he experienced a seizures attack three months after gene therapy. Generally, the adverse events and systemic complications were not observed.

After the injection on Feb 19, 2019, the patient's follow-up revealed recurrence of parietal focus on Dec 09, 2019; however, frontal focus had a slight and unremarkable enhancement. Until our last radiological follow-up (on Mar 18, 2020), frontal focus had no prominent recurrence and the size of the parietal focus increased and extended to the contralateral hemisphere through the corpus callosum (Fig. 2). Eventually patient has passed away on July 16, 2020 (PFS $=293$ days, OS $=657$ days).

\section{Discussion}


Suicide gene therapy as the most widespread and promising cancer gene therapy could be investigable intervention to reduce the burden of these tumors. Regarding the researches, it was highly suggested that approaching suitable results rely on promoting delivery system $(10,11)$. So we conducted our research with boosting the delivery system by using MSC and lentiviral vectors.

Furthermore, lots of factors and confounders affect the result of treatments in GBM clinical trial such as age, sex, karnofsky score, and genome of the patients (12). To the best of our knowledge, in our research we compared the pattern of growth of two foci in one patient for the first time. So we decreased some of confounding factors and simulate the response of tumor more precisely.

Studies on suicide gene therapy in the gliomas mostly applied the HSV-TK, carboxy-esterase rabbit (rCE) / CPT-1, and cytosine daemane / 5-fluorocytosine $(C D / 5 F C)(13,14)$. Among the viral vectors, retroviruses and adenoviruses are two suitable carriers mostly used as a delivery vehicle in both primary and recurrent glioblastoma tumors $(15,16)$. Rainov et al. described an open-label, randomized, phase III clinical study of retrovirus-mediated HSV-TK gene transfer with subsequent intravenous ganciclovir administration in primary GBM (15).

A large number of studies have demonstrated the tropism of various stem cells home on the tumor. Intrinsic tropism of MSCs to tumor tissue and their low immunogenicity indicates that utilizing MSCs could solve the low therapeutic efficiency in GBM standard treatment $(17,18)$. Some studies have shown the ability and efficiency of MSCs as a vehicle for the delivery of anti-cancer compounds into some tumor models like GBM, non-small-cell lung cancer, and breast cancer (19).

There was indication that utilizing MSCs as a vehicle could harbour more efficient outcomes. Previous clinical trials have been emphasized by many on increasing the delivery efficiency of genes in the tumor site. A large number of studies have demonstrated the tropism of various stem cells to the cancer site. Although these studies focused on genetically engineered stem cells, their delivery capabilities also had encouraging results $(17,18,20,21)$. Some clinical studies have shown the ability and efficiency of these cells to transmit genes to some tumor models like GBM, non-small-cell lung cancer, and breast cancer (17, $22,23)$. However, its clinical investigation is limited to just advanced gastrointestinal tumors (19). Indeed, clinical studies on MSCs and tumors as well as viruses have been restricted due to safety concerns, which have constrained the scope of these studies. Einem, J. et al.'s work which was the first clinical study to use genetically engineered MSCs in humans. This single-arm phase I/II study was carried out to evaluate the safety and efficacy of genetically modified autologous MSCs as delivery vehicles for cellbased gene therapy in the treatment of advanced, recurrent or metastatic gastrointestinal or hepatopancreatobiliary adenocarcinoma (TREAT-ME1 study). The first phase of the study, performed on six patients, indicated that the use of their product was safe during three cycles with different concentrations plus ganciclovir. In the second phase, a clinical study was conducted on 16 patients in two groups: a patient with adenocarcinoma from the digestive system that qualified for surgery with prior neoadjuvant treatment and a patient with advanced adenocarcinoma $(24,25)$. 


\section{Conclusion}

The gliomatous focus that undergone transplantation had a different pattern of growth and recurrence compared to a non-treated one. This conclusion might be somewhat restricted because of the limitations of the case report.

\section{Abbreviations}

Glioblastoma Multiform (GBM); Mesenchymal Stem Cells (MSCs); herpes simplex virus thymidine kinase (HSV-TK); Karnofsky Performance Score (KPS); Human embryonic kidney (HEK); vesicular stomatitis viruses (VSVs); glycoprotein (G); multiplicity of infection (MOI); criteria for adverse events (CTCAE); Response Evaluation Criteria in Solid Tumors (RECIST); carboxy-esterase rabbit (rCE); CPT-1, and cytosine daemane; 5-fluorocytosine (CD / 5FC).

\section{Declarations}

\section{Data availability}

In case of any request for the data, the demands, reasons, and Curriculum Vitae of the applicant will be reviewed and at the discretion of the corresponding author, the data will be provided to the applicant.

\section{Ethics approval and consent to participate}

This study has been approved by the Ethics committee in the Medical Research of the Shahid Beheshti University of Medical Sciences (Ethics code: IR.SBMU.REC.1396.224) on 2017-06-07. Informed consent form of the study has been reviewed by the mentioned ethics committee and the patient signed it before intervention.

\section{Trial registration}

IRCT20200502047277N2. Registered 10 May 2020 - Retrospectively registered, https://eng.irct.ir/trial/48110

\section{Consent for publication}

Not applicable.

\section{Funding}

This research has been funded by Shahid Beheshti University of Medical Sciences 
We declare no competing interests.

\section{Author contribution:}

SY: Project Designing and conducting clinical procedures; MA: Writing the manuscript and performing literature review; MG: Editing the manuscript and designing the follow up; GS: Conducting the laboratory procedures; FR: Conducting the laboratory procedures; $\mathrm{MH}$ : Conducting the laboratory procedures; $\mathrm{MY}$ : Following up the patients; AZ: Supervising the clinical procedures; MS: Supervising the laboratory procedures.

\section{Acknowledgements}

None.

\section{References}

1. Abou-El-Ardat K, Seifert M, Becker K, Eisenreich S, Lehmann M, Hackmann K, et al. Comprehensive molecular characterization of multifocal glioblastoma proves its monoclonal origin and reveals novel insights into clonal evolution and heterogeneity of glioblastomas. Neuro-Oncology. 2017;19(4):546-57.

2. Thomas RP, Xu LW, Lober RM, Li G, Nagpal S. The incidence and significance of multiple lesions in glioblastoma. Journal of neuro-oncology. 2013;112(1):91-7.

3. Dixit K, Kumthekar P. Gene Delivery in Neuro-Oncology. Current Oncology Reports. 2017;19(11).

4. Ishii-Morita H, Agbaria R, Mullen C, Hirano H, Koeplin D, Ram Z, et al. Mechanism of 'bystander effect'killing in the herpes simplex thymidine kinase gene therapy model of cancer treatment. Gene Ther. 1997;4(3):244-51.

5. Nowakowski A, Andrzejewska A, Janowski M, Walczak P, Lukomska B. Genetic engineering of stem cells for enhanced therapy. Acta Neurobiol Exp (Wars). 2013;73(1):1-18.

6. Studeny M, Marini FC, Dembinski JL, Zompetta C, Cabreira-Hansen M, Bekele BN, et al. Mesenchymal stem cells: Potential precursors for tumor stroma and targeted-delivery vehicles for anticancer agents. J Natl Cancer Inst. 2004;96(21):1593-603.

7. Barry FP, Murphy JM. Mesenchymal stem cells: Clinical applications and biological characterization. International Journal of Biochemistry Cell Biology. 2004;36(4):568-84.

8. Yazdani SO, Hafizi M, Zali A-R, Atashi A, Ashrafı F, Seddighi A-S, et al. Safety and possible outcome assessment of autologous Schwann cell and bone marrow mesenchymal stromal cell cotransplantation for treatment of patients with chronic spinal cord injury. Cytotherapy. 2013;15(7):782-91.

9. Eisenhauer EA, Therasse P, Bogaerts J, Schwartz LH, Sargent D, Ford R, et al. New response evaluation criteria in solid tumours: revised RECIST guideline (version 1.1). European journal of cancer. 2009;45(2):228-47. 
10. Bonini C, Bondanza A, Perna SK, Kaneko S, Traversari C, Ciceri F, et al. The Suicide Gene Therapy Challenge: How to Improve a Successful Gene Therapy Approach. Mol Ther. 2007;15(7):1248-52.

11. Fillat C, Carrió M, Cascante A, Sangro B. Suicide gene therapy mediated by the Herpes Simplex virus thymidine kinase gene/Ganciclovir system: fifteen years of application. Curr Gene Ther. 2003;3(1):13-26.

12. Patil CG, Yi A, Elramsisy A, Hu J, Mukherjee D, Irvin DK, et al. Prognosis of patients with multifocal glioblastoma: a case-control study. 2012;117(4):705.

13. Choi SA, Lee JY, Wang KC, Phi JH, Song SH, Song J, et al. Human adipose tissue-derived mesenchymal stem cells: Characteristics and therapeutic potential as cellular vehicles for prodrug gene therapy against brainstem gliomas. Eur J Cancer. 2012;48(1):129-37.

14. Fei S, Qi X, Kedong S, Guangchun J, Jian L, Wei Q. The antitumor effect of mesenchymal stem cells transduced with a lentiviral vector expressing cytosine deaminase in a rat glioma model. J Cancer Res Clin Oncol. 2012;138(2):347-57.

15. Rainov NG. A phase III clinical evaluation of herpes simplex virus type 1 thymidine kinase and ganciclovir gene therapy as an adjuvant to surgical resection and radiation in adults with previously untreated glioblastoma multiforme. Hum Gene Ther. 2000;11(17):2389-401.

16. Ram Z, Culver KW, Oshiro EM, Viola JJ, Devroom HL, Otto E, et al. Therapy of malignant brain tumors by intratumoral implantation of retroviral vector-producing cells. Nat Med. 1997;3(12):1354-61.

17. Hoyos V, Del Bufalo F, Yagyu S, Ando M, Dotti G, Suzuki M, et al. Mesenchymal Stromal Cells for Linked Delivery of Oncolytic and Apoptotic Adenoviruses to Non-small-cell Lung Cancers. Mol Ther. 2015;23(9):1497-506.

18. Metz MZ, Gutova M, Lacey SF, Abramyants Y, Vo T, Gilchrist M, et al. Neural stem cell-mediated delivery of irinotecan-activating carboxylesterases to glioma: implications for clinical use. Stem Cells Transl Med. 2013;2(12):983-92.

19. Westphal M, Ylä-Herttuala S, Martin J, Warnke P, Menei P, Eckland D, et al. Adenovirus-mediated gene therapy with sitimagene ceradenovec followed by intravenous ganciclovir for patients with operable high-grade glioma (ASPECT): A randomised, open-label, phase 3 trial. The Lancet Oncology. 2013;14(9):823-33.

20. Kim SK, Kim SU, Park IH, Bang JH, Aboody KS, Wang KC, et al. Human neural stem cells target experimental intracranial medulloblastoma and deliver a therapeutic gene leading to tumor regression. Clin Cancer Res. 2006;12(18):5550-6.

21. Grisendi G, Bussolari R, Cafarelli L, Petak I, Rasini V, Veronesi E, et al. Adipose-derived mesenchymal stem cells as stable source of tumor necrosis factor-related apoptosis-inducing ligand delivery for cancer therapy. Cancer Res. 2010;70(9):3718-29.

22. Rath P, Shi H, Maruniak JA, Litofsky NS, Maria BL, Kirk MD. Stem cells as vectors to deliver HSV/tk gene therapy for malignant gliomas. Curr Stem Cell Res Ther. 2009;4(1):44-9.

23. O'Brien KP, Khan S, Gilligan KE, Zafar H, Lalor P, Glynn C, et al. Employing mesenchymal stem cells to support tumor-targeted delivery of extracellular vesicle (EV)-encapsulated microRNA-379. Oncogene. 
2018;37(16):2137-49.

24. von Einem JC, Peter S, Günther C, Volk H-D, Grütz G, Salat C, et al. Treatment of advanced gastrointestinal cancer with genetically modified autologous mesenchymal stem cells - TREAT-ME-1 a phase I, first in human, first in class trial. Oncotarget. 2017;8(46).

25. von Einem JC, Guenther C, Volk H-D, Grütz G, Hirsch D, Salat C, et al. Treatment of advanced gastrointestinal cancer with genetically modified autologous mesenchymal stem cells: Results from the phase 1/2 TREAT-ME-1 trial. Int J Cancer. 2019;145(6):1538-46.

\section{Figures}
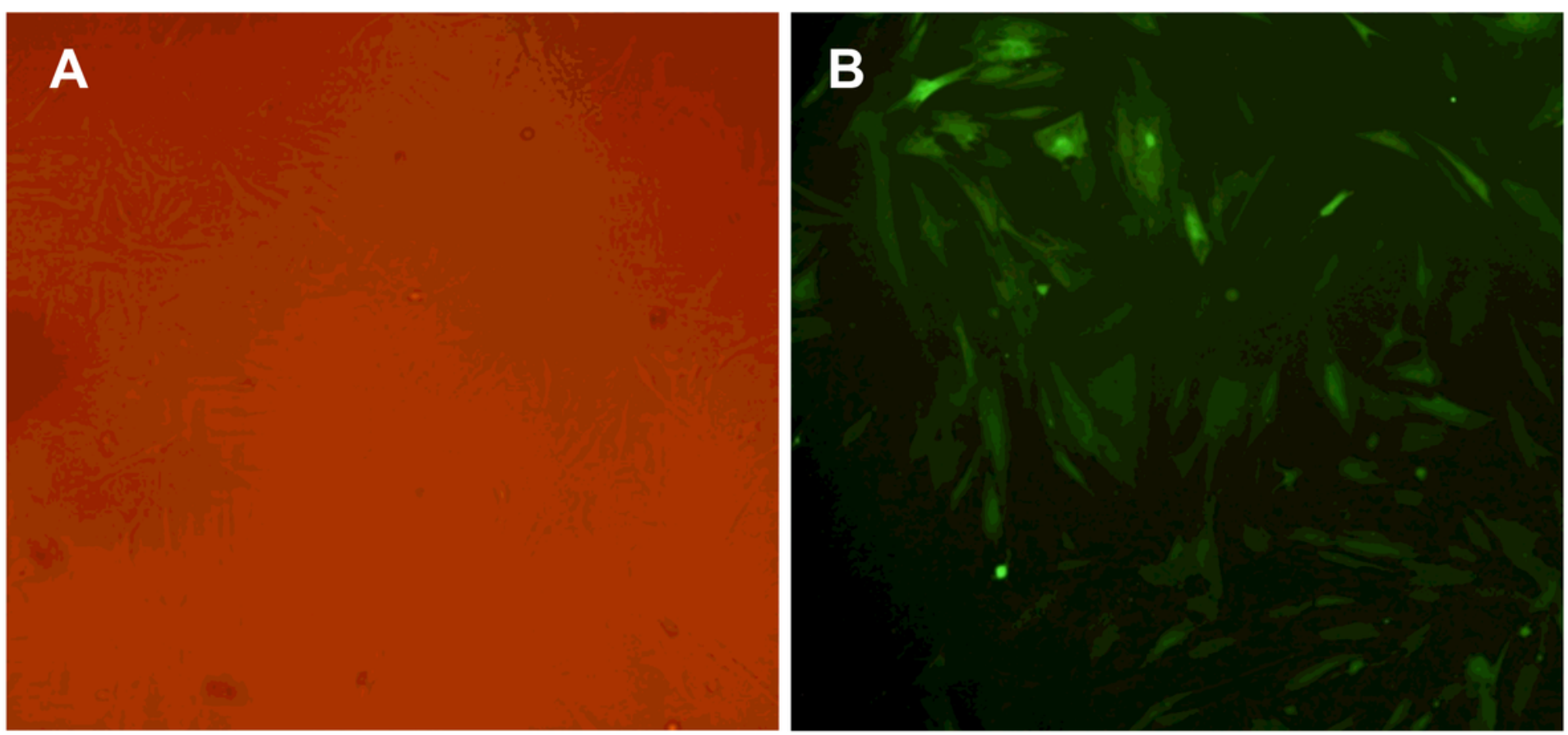

\section{Figure 1}

The image above shows the Mesenchymal Stem Cells transduced with an invert microscope (X10), which shows $A$ ) visible light and $B$ ) fluorescent light. 

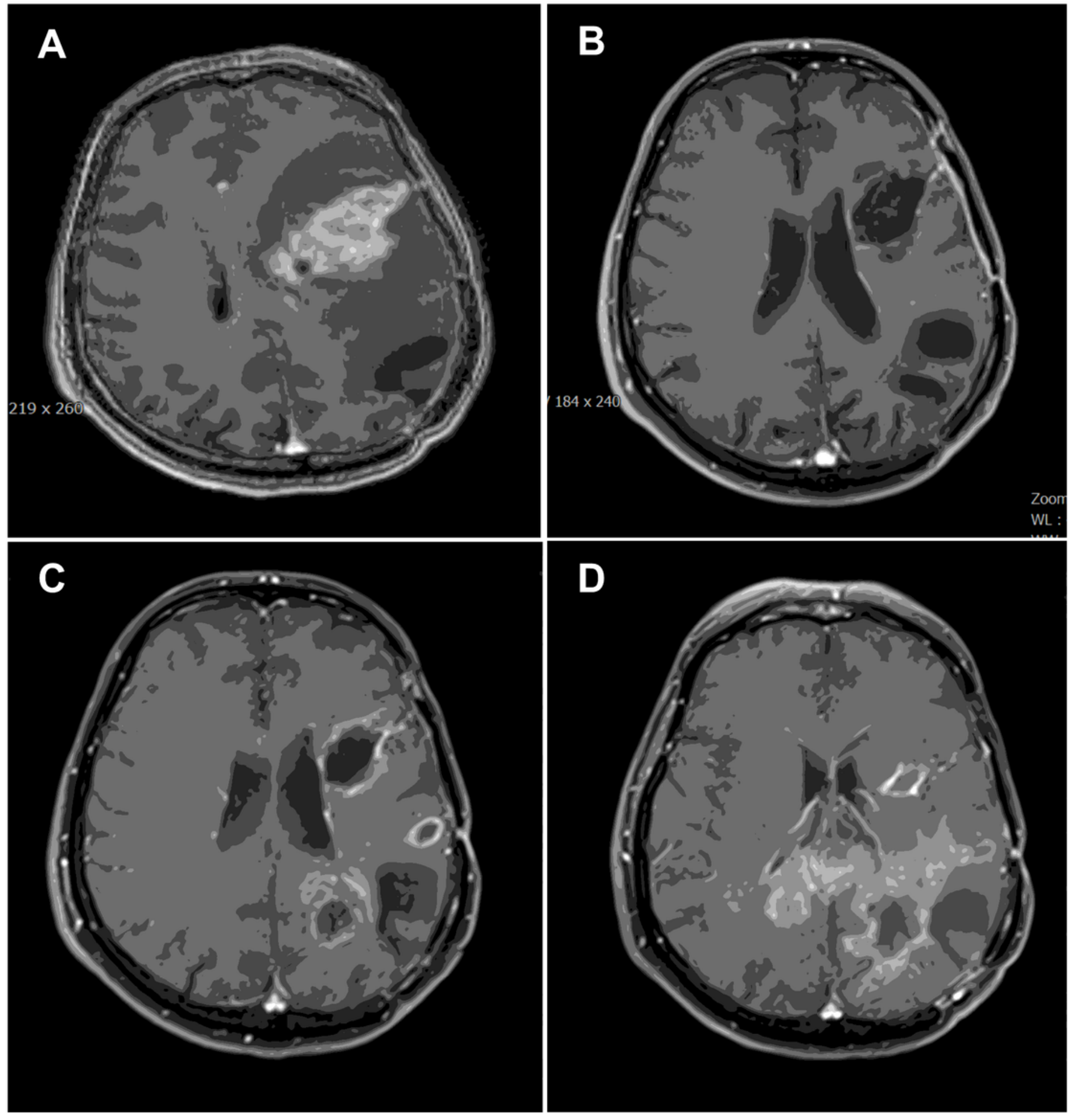

\section{Figure 2}

Magnetic Resonance Imaging of the patient at: A) November 2018 which shows left frontal lesion and evidence of craniotomy related to previous surgery for parietal lesion one month ago; B) February 2019 with no obvious evidence of recurrence at the time of injection in the frontal site; C) December 2019 which shows first evidence of recurrence of tumor mostly at parietal site; D) March 2020, left frontal 
focus had no prominent recurrence, however, the size of the left parietal focus increased and extended to the contralateral hemisphere through the corpus callosum. 